\title{
Texture discrimination and unit recordings in the rat whisker/barrel system
}

\author{
Thomas Prigg $^{\mathrm{a}}$, Daniel Goldreich ${ }^{\mathrm{a}, 1}$, George E. Carvell ${ }^{\mathrm{b}}$, Daniel J. Simons ${ }^{\mathrm{a}, *}$ \\ a Department of Neurobiology, University of Pittsburgh School of Medicine, Pittsburgh, PA 15261, USA \\ ${ }^{\mathrm{b}}$ Department of Physical Therapy, University of Pittsburgh, Pittsburgh, PA, USA
}

Received 22 July 2002; accepted 21 September 2002

\begin{abstract}
We have developed a semi-automated technique for acquiring neurophysiological data during whisker-based tactile discriminative behavior. Water-deprived, blindfolded rats are tethered by means of a harness vest that permits them to contact a rough ( $250 \mu \mathrm{m}$ grooves) or smooth discriminandum with only their vibrissae. Discriminanda are mounted on a motor-driven carousel, and the rat indicates its choice (rough, smooth) by licking either a right or left water port located near the carousel. A narrow light beam detects general proximity of the animal's nose to the discriminandum, although actual whisker contact is monitored by a SuperVHS camera and measured offline using fieldby-field videographic analysis. Rats can be trained within 3-6 weeks at which time they perform $100-150$ trials/day at a level of $80 \%$ correct. Unit recording from the somatosensory cortex reveals that neurons increase their firing upon whisker contact of a discriminandum and that firing remains elevated during several hundred milliseconds of ongoing contact, even with the smooth surface. Nevertheless, despite the animal's ability to distinguish the rough and smooth surfaces, overall neuronal firing rates were indistinguishable for the two surfaces. In some cases, temporal firing patterns differed, although not in a consistent way across recording sites.
\end{abstract}

(C) 2002 Published by Elsevier Science Inc.

Keywords: Discrimination; Whisker/barrel system; Rat

\section{Introduction}

The rodent somatosensory system is highly specialized for processing fine tactile information acquired by the array of vibrissae, or whiskers, on the facial mystacial pad. Rats and other rodents actively sweep their vibrissae in a rhythmic forward and backward motion, called whisking, to locate and distinguish objects in the animal's immediate sensory environment [1]. Psychophysical studies of trained rats have demonstrated that animals using only their whiskers can reliably detect small differences in surfaces textures at a level comparable to human and nonhuman primates using their fingertips [2]. Anatomic and physiologic investigations reveal remarkable specialization in central nervous system structures that process and transmit whisker-based tactile information to the primary somato-

\footnotetext{
* Corresponding author. Tel.: +1-412-648-9442; fax: +1-412-6481441.

E-mail address: cortex@pitt.edu (D.J. Simons).

${ }^{1}$ Present address: Department of Occupational Therapy, Rangos School of Health Sciences, Duquesne University, Pittsburgh, PA 15282, USA.
}

sensory cortex. There, aggregations of layer IV neurons, called "barrels," are related one-to-one to individual whiskers on the contralateral mystacial pad [3]; these neuronal networks, or modules, transform afferent information from the somatosensory thalamus and distribute the transformed signal to other layers of the cortical column for further processing and integration [4]. In spite of our increasing understanding of whisker-related microcircuits in the brain, remarkably little is known about mechanisms of information processing during normal tactile behavior. This reflects in large part on an absence of methodologies that permits detailed examination of neural activity during discriminative whisking behavior.

Our previous studies of vibrissal-based texture discrimination employed a gap-jumping paradigm. In a forced, twochoice test, blindfolded rats were trained to stretch across a gap to palpate rough or smooth surfaces with their vibrissae and then jump from the start platform onto the reward platform. While behaviorally manageable, the gap-jumping paradigm was largely unsuitable for obtaining neurophysiological unit recordings. Because the task was physically demanding, animals would perform only a limited number of trials per day, and continual experimenter intervention 
was required to operate the apparatus. Here we describe a technique for semi-automated testing that does not require gap jumping or extensive movement. Animals can perform large numbers of trials during a single testing session, permitting acquisition of neurophysiological data while the animal is engaging in a discriminative touch task.

\section{Materials and methods}

\subsection{Subjects}

One adult, female Sprague-Dawley rat (Hilltop Laboratory Animals, Scottsdale, PA) underwent two aseptic surgeries while anesthetized with halothane. During the first procedure, an acrylic "cap" was affixed to the skull using small stainless steel screws. The cap was used to secure an electrical connector and screw-mounts for attaching removable blindfolds (see Ref. [2]). Two to six weeks later, a second surgery was performed in order to create a small craniectomy for access to the whisker representation in the barrel cortex and to attach a miniature microdrive to the dental acrylic appliances (see Ref. [5]). Torbugesic ( $2 \mathrm{mg} / \mathrm{kg}$ sc) was administered postoperatively, and for the next 3 days the animal was given two crushed baby aspirin in its drinking water.

\subsection{Training apparatus and procedure}

The animal was trained on a discrimination task developed for other rats, as follows. For behavioral training/ testing, rats were water-deprived $22 \mathrm{~h} /$ day for 5 days/week. Animals received water during the training session and were allowed unlimited access to water for 20-30 min following each session. The condition of the animal was assessed daily, and all subjects remained vigorous and alert and gained weight throughout the course of study. Rats were trained to discriminate between two surfaces using only their mystacial vibrissae. At the beginning of each session, the animal was blindfolded and fitted with a body harness and connecting tether. The length of the tether was adjusted so that animals could contact the discriminandum only with their outstretched (protracted) whiskers. Discriminanda were 3 in. long by 1 in. diameter plastic cylinders (Delrin, Dupont). One cylinder consisting of stock material was smooth, whereas the other had grooves of equal width, depth and spacing of $250 \mu \mathrm{m}$. Previous studies have shown that rats readily distinguish between these two surfaces $[2,6]$.

Discriminanda were mounted on a motor-driven carousel, and at the onset of each trial, cued by white noise, either the rough or the smooth surface, selected randomly, was presented to the animal (Fig. 1). The rat indicated its

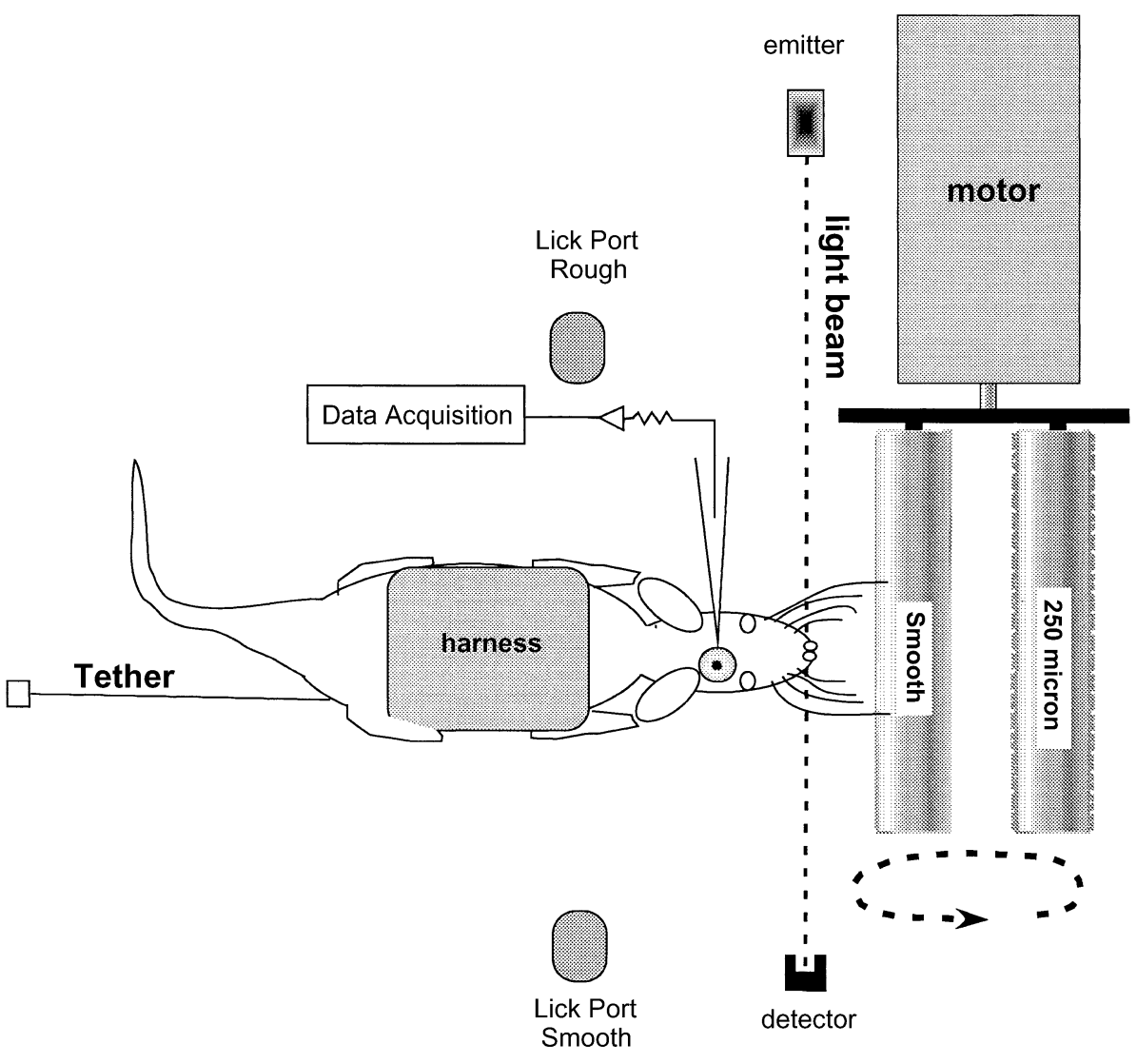

Fig. 1. Schematic of training apparatus. Not drawn to scale. 
response (smooth, rough) by licking either a right or left water port placed near the carousel. A narrow light beam projected in front of the cylinder detected proximity of the animal's nose to the discriminandum and was used to initiate data collection during electrophysiological recordings. Palpation of the discriminandum by the whiskers was continuously monitored using a SuperVHS camera and viewed using a video cassette recorder that split each frame into two separate fields spaced $16.67 \mathrm{~ms}$ apart. A MacIntosh computer, running Labview (National Instruments, Austin, TX), controlled the behavioral apparatus, logged animal performance and acquired electrophysiological data.

Training consisted of five stages. During Stage 1, in the first 2 weeks, the animals were acclimated to handling by the trainer and to wearing the tether. During Stage 2, which lasted 1-3 days, rats learned to associate the training apparatus with water availability. For $1 \mathrm{~h}$ daily they were tethered inside the training apparatus and given free access to water in a centrally located dish. In Stage 3, also lasting 1-3 days, water was available only from the lick-ports; water availability, which was signaled by a click sound produced by the solenoid-activated delivery valve, was randomized between the two ports. In Stage 4, discriminanda were introduced using the motorized carousel. White noise cued the presentation of a discriminandum, and when the animal interrupted the light beam, water, accompanied by an explicit click, was delivered to the appropriate lick port. The tether was used to prevent the rat from touching the discriminanda with its nose or forepaws. Stage 4 lasted 2-3 days with 100-200 trials/day. In Stage 5, water reward $(\sim 0.2 \mathrm{ml}$ each) was made contingent on the animal selecting the correct lick port, which was detected electronically by means of a tongue-to-ground contact circuit (Coulbourn Instruments). Incorrect decisions were indicated by an error tone, followed by a timeout of $\sim 4 \mathrm{~s}$; correct trials were followed almost immediately by the start of the next trial. Animals remained on task as long as they demonstrated interest in it (100-200 trials) during which time they consumed as much as $20-25 \mathrm{ml}$ of water, depending on the number of rewarded trials. Following the training session, rats drank additional water within a $20-30 \mathrm{~min}$ period during which they ceased drinking. Total water intake was at least $40 \mathrm{ml} /$ day.

\subsection{Electrophysiological recordings and data analysis}

In one animal, we recorded unit activity during the discrimination task. After it had attained a criterion performance of $90 \%$ correct, the animal underwent the second surgical procedure described above and in detail in Ref. [7]. A recording microelectrode was positioned in order to monitor activity from the representation of whiskers in the middle of the mystacial pad. Unit recording data were obtained using epoxylite-coated platinum-iridium electrodes (impedances 3-5 $\mathrm{M} \Omega$ at $1000 \mathrm{~Hz}$ ) over multiple daily sessions, as described previously [5]. Records were processed offline to isolate the largest amplitude spikes, which we estimate consisted of discharges from 1 to 3 units. Unit activity was related to palpation of a discriminandum using the proximity-detecting light beam and field-by-field video analysis. For each trial, the time (video field) of first contact was noted, and unit activity was measured beginning from $100 \mathrm{~ms}$ prior to contact and for the duration of the palpation period, which varied from 150 to $500 \mathrm{~ms}$. The average firing rate during each of these epochs was calculated. At the end of each recording session, the electrode was withdrawn above the brain surface and readvanced to the vicinity of layer IV prior to the next session. On the final day of recording, a microlesion was produced. The rat was deeply anesthetized with pentobarbital sodium (100 $\mathrm{mg} / \mathrm{kg}$ ip) and perfused for cytochrome oxidase and Nissl taining. The cortex was sectioned at $60 \mu \mathrm{m}$, and the localizations of recording sites to barrel cortex were confirmed histologically.

\section{Results}

\subsection{Unit activity during palpation of textured surfaces}

Just prior to the start of a recording session, the microelectrode was advanced until cortical units were encountered that responded to manual deflections of one or more whiskers located near the center of the mystacial pad. Fig. 2 shows a representative trace obtained during a single discrimination trial. The heavy horizontal bar beneath the trace indicates the time period during which the animal was

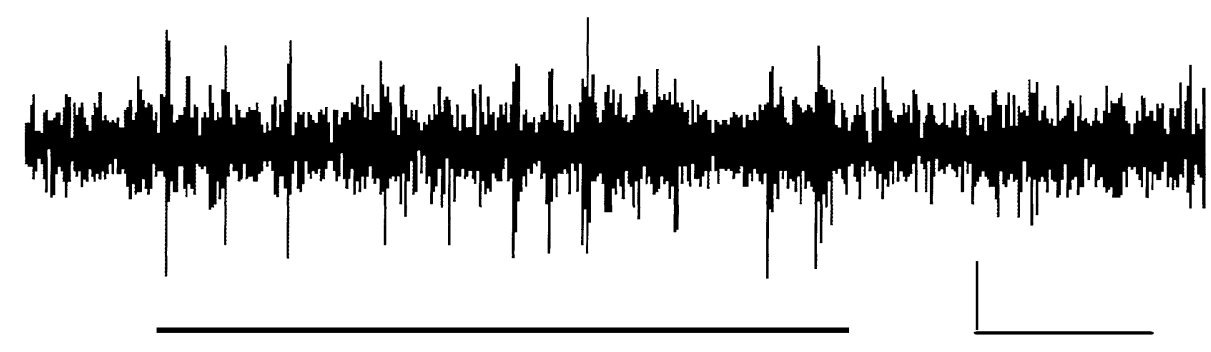

Fig. 2. Example of unit recordings. Solid line beneath trace denotes period when whiskers were contacting discriminandum, determined from field-by-field videography. Scale bar at right: $100 \mathrm{~ms}, 75 \mu \mathrm{V}$. 
contacting the discriminandum with its whiskers; this period is preceded by the final $10 \mathrm{~ms}$ of the animal's approach to the surface. Unit activity increased upon surface contact and remained elevated during the $\sim 400 \mathrm{~ms}$ of contact. Immediately afterwards, the animal turned towards the water port, and overall unit firing decreased.

Spike counts were obtained for trials in which whisker contact with a discriminandum was maintained for a period $>200 \mathrm{~ms}$, as determined from the video recordings. As a further confirmation that the animal was palpating the surface, data analyses were restricted to trials in which the animal made a correct decision. The data set consists of 234 smooth trials and 182 rough trials distributed over 13 recording sessions. Fig. 3 shows the average peri-contact time histogram $(n=13)$ in which unit activity is aligned temporally with the estimated time first whisker contact with the discriminandum; time of contact is set at the 100th 1-ms bin. Initial contacts were estimated from field-by-field analyses of video images collected at a resolution of 16.67 ms per field. For both rough and smooth surfaces, there is a notable increase in the population's response upon initial contact. Interestingly, unit activity remains elevated even when whiskers were contacting the smooth discriminandum, reflecting perhaps the bending of the whiskers. There is no obvious difference in the peri-stimulus time histograms (PSTHs) for the rough and smooth surfaces. Mean spike

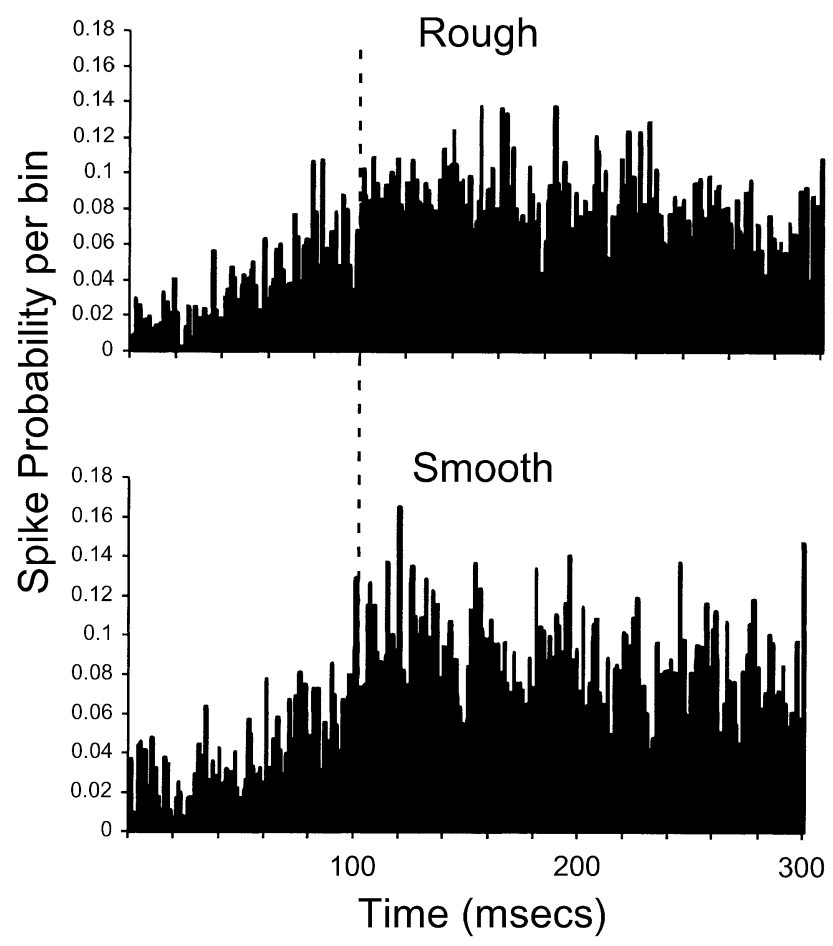

Fig. 3. Peri-contact time histograms constructed for all units/recording sites. Height of each bar is the probability of a spike occurring in that 1-ms bin. Dotted vertical line indicates approximate time of first whisker contact, determined videographically. The rough surface had grooves of equal width, depth and spacing of $250 \mu \mathrm{m}$; the smooth surface had no grooves.

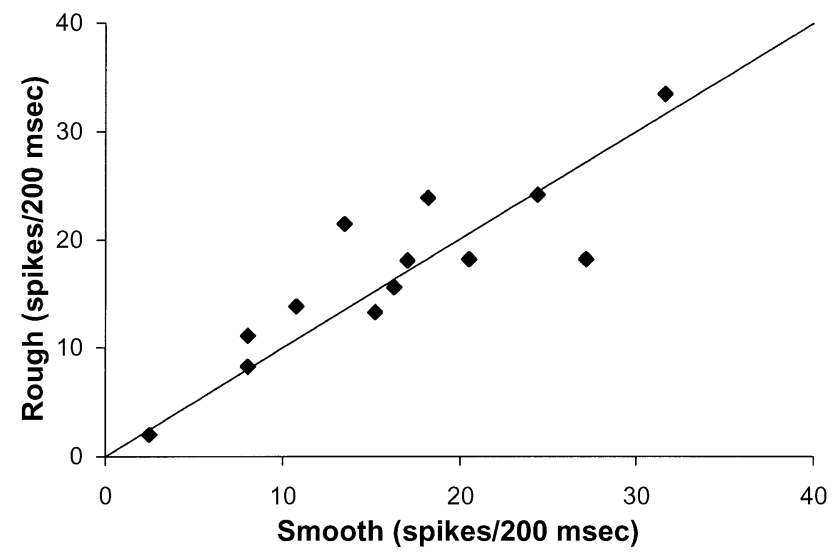

Fig. 4. Scatterplot showing mean number of spikes per trial during the first $200 \mathrm{~ms}$ of whisker contact of smooth and rough surfaces at each of 13 different recording sites within the somatosensory "barrel" cortex. Diagonal line denotes a hypothetical 1:1 relationship.

counts per $200 \mathrm{~ms}$, shown in the scatterplot of Fig. 4, did not differ statistically (rough: $17.46 \pm 8.45$ S.D.; smooth: $16.79 \pm 7.89$ S.D., two-tailed paired $t$ test, $P=.60)$. Similar findings were obtained for the first $100 \mathrm{~ms}$ of contact.

We also examined interspike interval distributions to determine whether neuronal firing patterns differed for the rough vs. smooth surfaces. Again, no differences were in found in any of the 13 recording sites (Wilcoxon rank sum test with correction for multiple comparisons).

\section{Discussion}

The present results extend previous findings showing that rats can use their whiskers to distinguish between surfaces having subtly different textures and shape $[2,6,8,9]$. The behavioral training methodology described here offers several advantages to earlier approaches that required considerable handling of the animal between trials and that, in the case of gap jumping, limited acquisition of data from large numbers of trails per recording session. The semi-automated apparatus permits excellent synchronization of behavioral and neurophysiological data, provided that the onset and duration of whisker contact can be accurately measured. We found, however, that proximity detection by light beams near the discriminandum surface is insufficient for ensuring that whiskers maintain surface contact during a trial. This necessitated the use of offline, labor-intensive videographic analyses by trained observers, using images having limited temporal and spatial resolution. Moreover, high illumination levels needed for the videography require that the rats be blindfolded, requiring more extended periods of acclimation and physical restrictions in mounting recording instrumentation to the animal's head.

Previous neurophysiological studies in nonhuman primates have provided evidence that roughness is encoded in the somatosensory cortex by changes in mean neuronal 
firing rates $[10,11]$. Compared to the textures used in those studies, the rough surface used here had a much finer scale. Lesions of the somatosensory "barrel" cortex led to deficits in vibrissal-based texture discrimination [12], and we expected that differences in the firing of cortical neurons would be small but detectable, with at least some neurons firing at higher average rates when the rat palpated the rough compared to the smooth surface. We found, however, that neither overall firing rates nor temporal discharge patterns are robust indicators of the rough and smooth surfaces. Our findings may reflect the small and perhaps heterogenous sample of neurons and/or our inability to correlate precisely whisker contact and neural response. Alternatively, the encoding of subtly different surfaces, such as those used here, may involve conjoint firing of multiple neurons within select populations [13]. Such neural correlates of fine texture discrimination may be evident only when the activities of many networked neurons are recorded simultaneously. Because of substantial limitations in the use of videography for monitoring whisking, the practicality of analyzing such large data sets will require the use of automated procedures for directly and precisely measuring the time of surface contact by the whiskers.

\section{Acknowledgements}

We thank Randy Bruno for assistance with data analysis. Supported by NSF IBN-9209490 and NIH NS19950.

\section{References}

[1] Welker WI. Analysis of sniffing of the albino rat. Behaviour 1964;22: $223-44$.

[2] Carvell GE, Simons DJ. Biometric analyses of vibrissal tactile discrimination in the rat. J Neurosci 1990;10:2638-48.
[3] Woolsey TA, Van der Loos H. The structural organization of layer IV in the somatosensory region (SI) of mouse cerebral cortex. Brain Res 1970;17:205-42.

[4] Simons DJ. Response properties of vibrissa units in the rat SI somatosensory neocortex. J Neurophysiol 1978;41:798-820.

[5] Carvell GE, Miller SA, Simons DJ. The relationship of vibrissal motor cortex unit activity to whisking in the awake rat. Somatosens Mot Res 1996;13:115-27.

[6] Carvell GE, Simons DJ. Task- and subject-related differences in sensorimotor behavior during active touch. Somatosens Mot Res 1995; $12: 1-9$.

[7] Kelly MK, Carvell GE, Jobling J, Simons DJ. Sensory loss by selected whisker removal produces immediate disinhibition in the somatosensory cortex of behaving rats. J Neurosci 1999;19:9117-25.

[8] Brecht M, Preilowski B, Merzenich MM. Functional architecture of the mystacial vibrissae. Behav Brain Res 1997;84:81-97.

[9] Guic-Robles E, Valdivieso C, Guajardo G. Rats can learn a roughness discrimination using only their vibrissal system. Behav Brain Res 1989;31:285-9.

[10] Sinclair RJ, Burton H. Neuronal activity in the primary somatosensory cortex in monkeys (Macaca mulatta) during active touch on textured surface gratings: responses to groove width, applied force, and velocity of motion. J Neurophysiol 1991;66:153-69.

[11] Tremblay F, Ageranioti-Belanger SA, Chapman CE. Cortical mechanisms underlying tactile discrimination in the monkey: I. Role of primary somatosensory cortex in passive texture discrimination. $\mathrm{J}$ Neurophysiol 1996;76:3382-403.

[12] Guic-Robles E, Jenkins WM, Bravo H. Vibrissal roughness discrimination is barrel cortex-dependent. Behav Brain Res 1992;48:145-52.

[13] Darian-Smith I, Sugitani M, Heywood J, Karita K, Goodwin A. Touching textured surfaces: cells in somatosensory cortex respond both to finger movement and to surface features. Science 1982;218: 906-9. 